the Agency for Healthcare Research and Quality were judged to still be valid. ${ }^{13}$ Seven (41\%) of the CPGs were found to require a major update to reflect new evidence, and 6 CPGs were judged to require a "minor update." In other words, at the time of the report in September 2001, three quarters of the AHRQ guidelines needed updating.

In fact, there are at least 8 threats to the reliability and usefulness of information contained in CPGs and CPMs. First, bias and conflict of interest can undermine validity and reliability (value) of the clinical "evidence." Second, experts may fail to use sufficient rigor when interpreting the results of clinical interventions. Third, valid and reliable clinical evidence may not be reduced to a practical and useful CPG. Fourth, experts may not disclose conflict of interest and potential bias when preparing CPGs. Fifth and sixth, a useful and practical CPG may not be disseminated adequately or may be unavailable at the point of care. Seventh, the communication of the guideline may be ineffective or become lost in the increasing level of "noise" in information communicated to physicians. Eighth, even if readily available at the point of care, the clinician may reject or otherwise choose to ignore the $\mathrm{CPG}$ and the information it contains. All 8 barriers may contribute to nonrandom variation in clinical practice.

An online survey of about 300 internal medicine and family practice physicians conducted by Harris Interactive in mid-2001 for the Henry J. Kaiser Family Foundation found that only 19\% of these primary care physicians chose aspirin as the treatment of choice for the onset of stroke, $27 \%$ chose heparin, and about half opted for t-PA. According to clinical evidence, t-PA is the thirdline choice for acute stroke, while aspirin is first-line and heparin is second-line. Some of the gap in the use of CPGs is attributable to physician resistance to "cookbook medicine." Alan Muney, MD, chief medical officer for Oxford Health Plans (Trumbull, Connecticut) commented, "The shameful truth is that not all doctors adhere to evidence-based medical guidelines."14 "Shameful" might not be the best word to describe the failure by clinicians to use the available information since practicing clinicians point, fairly, to bias in the preparation of many CPGs and CPMs. There is also the matter of inadequate communication of the CPG to the practitioner. Amid an increasing barrage of data, practitioners could easily lose the significance of an otherwise important clinical guideline among the "noise" of data overload.

The matter of pseudo-evidence is also of serious concern and contributes to the problem of data overload and information underload. Calling data "evidence" does not make it so. Much of the literature contains expert opinion, not the results of randomized clinical trials. It has been said that one study, particularly a randomized, control group trial, is worth 1,000 expert opinions.

\section{Information Technology to Cross the Quality Chasm}

At year-end 1997, the Health Care Financing Administration, now the Centers for Medicare and Medicaid Services, proposed a maximum 2\% medication error rate for Medicare and Medicaid hospitals, and nurses would be required "to review drug orders for accuracy of the entire system before prescription drugs are administered."15 In 1998, a 15-month study of computerized physician-order entry at Brigham \& Women's Hospital found that medication errors were reduced by $55 \%$ when physicians were required to enter all drug orders by computer. ${ }^{16}$ While few would debate the need to improve quality by reducing medication error rates, there is debate about the magnitude of the problem.

More than 2 years later, General Motors Corp., IBM, AT\&T, General Electric, Boeing, and 91 other employers collaborated to form the Leapfrog Group, to, as its name implies, leapfrog over the present (slow) pace of quality improvement in health care. The Leapfrog Group in 2001 defined 3 basic ways to improve safety and quality of health care for at least the hospital component of the health system. The Leapfrog Group urged managed care organizations (MCOs) to contract with only those hospitals that (1) implemented a computerized order entry system by 2004 and (2) provided an ICU staffed full-time by an internist with specialty training and certification in intensive care medicine. Third, the Leapfrog Group suggested that MCOs should practice evidence-based medicine by sending patients only to hospitals with high volume and favorable outcomes. As for the chasm between current practice and the 3 quality standards: only $3.3 \%$ of hospitals reported operational physician electronic order entry systems, only $10 \%$ of hospitals surveyed had intensive-care specialists overseeing care in the ICU at least 8 hours per day, and only $12 \%$ of 250 hospitals surveyed by the Leapfrog Group in late 2001 met the standard of performing at least 500 coronary artery bypass graft procedures per year.

At a meeting on July 26, 2002, called by the U.S. Food and Drug Administration (FDA), hospital groups and patient safety advocates such as the Institute for Safe Medication Practices (ISMP) asked the FDA to require prescription drug manufacturers to apply bar codes to all drug packages, particularly unitdose packages. The promise is large for information technology (IT) to reduce the estimated 100,000 deaths annually and the estimated 770,000 medication errors that occur in U.S. hospitals each year. ${ }^{17}$ Yet, there are several factors that should affect expectations of the promise of IT to cross the quality chasm in medication errors. First, there is the absence of a single bar code standard. Second, even if there were bar codes on individual drug doses (unit dose packages), only 10\% to 15\% of hospitals had bar code readers at patient bedsides in mid-2002. Third, drug manufacturers had been producing fewer drugs in unitdose packages, due to the extra cost. Fourth, there is a question of the return-on-investment. Bar coding drugs and making bar code readers standard at hospital bedsides would cost $\$ 1.5$ billion or more, and the FDA estimate of the 770,000 annual hospital medication errors that are preventable is a wide range, from $28 \%$ to $95 \% .^{18}$ 
Lucian Leape, MD, a retired surgeon and professor at the Harvard School of Public Health, found wide discrepancies in hospital medication error rates, attributing the discrepancies to the method of data reporting rather than actual variation in incidence and outcomes, highlighting the fact that people who report high medication error rates are punished for these data. Leape found an error rate of $0.2 \%$ (2 per 1,000 charts) for selfreport methods, $0.7 \%$ for retrospective chart reviews, $3.8 \%$ for computer screening, $6.5 \%$ for daily chart reviews, and $10.0 \%$ for computer screening combined with daily chart review. He concluded, "If you are relying on incident reports, you are missing $95 \%$ of them."

So, it would seem that (a) everybody agrees that medical errors should not occur, (b) the scope of the problem is not well-defined quantitatively, and (c) we have solutions for reducing medication errors but determining the return-on-investment from these solutions will be difficult. Brent James, MD, a well-recognized leader and teacher in health care quality improvement, has observed for several years that the key to reducing systematic variation in health care delivery involves "making it easy to do it right." This perspective involves making key process steps routine and unavoidable, such as the application of IT to the hospital discharge process. A randomized trial of a computerized clinical-information system that generated preventive care reminders at the point of patient discharge increased significantly the use of subcutaneous heparin for patients at risk for venous thromboembolism, instructions to take aspirin for patients hospitalized for acute myocardial infarction, and the use of pneumococcal or influenza vaccine for eligible patients. ${ }^{19}$ James observed that "studies of clinical errors have identified a series of human limitations that lead to predictable failures of the health care delivery system. ${ }^{.20}$ In this issue of the Journal, James elaborates on his perspectives of the quality chasm in health care. ${ }^{21}$ His perspectives are important since his work on the Institute of Medicine "Chasm" report is almost incidental to his efforts over the last 20 years to improve health care delivery by teaching others the methods to apply analytical thinking and statistical tools to the study of the processes of care, measuring outcomes, and reducing nonrandom variation in clinical practice.

\section{Benefit Maximums Versus Drug Benefit Needs for Medicare Beneficiaries}

Annual dollar maximum limits for Medicare+Choice prescription drug benefits are common, an average \$1,149 in 1997 and as low as $\$ 600$ per year. By CY 2000, 38\% of Medicare+Choice members with prescription drug benefits had an annual maximum of $\$ 750$ or less. Data from the Kaiser Family Foundation also show that $13 \%$ of Medicare beneficiaries spent $\$ 2,000$ or more on prescription drugs in CY 2001, accounting for 52\% of total prescription drug spending for Medicare beneficiaries. ${ }^{22}$ Spending of $\$ 1,000$ or more was found among $28 \%$ of Medicare beneficiaries and accounted for $76 \%$ of total expenditures for prescription drugs. Yet, an amazing $17 \%$ of Medicare beneficiaries had no (\$0) spending on prescription drugs in CY 2001. A previous study in the Journal reported that for 2 cohorts of patients in a Medicare+Choice population, one using only community pharmacy services and the other using only mail-service pharmacy, only $0.18 \%$ (26 members) and $0.01 \%$ (2 members) respectively, reached their maximum drug benefi, which ranged from $\$ 500$ to $\$ 1,600$ in $1998 .^{23}$ These data are difficult to reconcile.

Survey data from 10,927 noninstitutionalized seniors in 8 geographically diverse states in 2001 showed that $35 \%$ of seniors with drug coverage under a Medigap policy, $25 \%$ of seniors enrolled in state pharmacy assistance programs, and 19\% of seniors in Medicare HMOs spent at least $\$ 100$ per month $(\$ 1,200$ per year) on prescriptions in 2001. ${ }^{24}$ Medicare HMOs were important sources of drug coverage for seniors in California (30\%) and Colorado (24\%) but were less important in other states, ranging from a low of $7 \%$ in Illinois to $14 \%$ in Pennsylvania.

In this issue, Cox and Henderson ${ }^{25}$ report that 24\% of the respondents in their survey of Medicare+Choice members (with limited prescription drug benefits) did not know if they had exceeded their annual drug benefit maximum in the preceding year (CY 2000), and 46\% did not know (accurately) the dollar amount of their annual benefit maximum ("cap"). This study did not link the survey responses to the demographics and drug benefits of the Medicare+Choice members. This link would have helped answer questions that arise from efforts to interpret the survey results. And, the fact that $24 \%$ of Medicare+Choice members reported that they were not aware of whether or not they had exhausted their prescription drug benefits may not be surprising in the context of the statistic that $17 \%$ of Medicare beneficiaries had no $(\$ 0)$ expenditures for prescription drugs in CY 2001.

The study by Cox and Henderson also did not include analysis of the reported behaviors compared to actual experience, as measured by drug claims in the database. This assessment would have provided more insight into this important question of the effects of Medicare+Choice annual maximum benefits on the use of prescription drugs by type (e.g., generic drugs versus brand drugs, antihypertensives versus drugs for heartburn). Also left to other researchers is evaluation of the qualitative and quantitative time curve to exhaustion of benefits; i.e., the percentage of beneficiaries who exhaust benefits in 90 days, 120 days, etc. Readers should also note that the work by Cox and Henderson did not include Medicare+Choice annual maximum benefits applied quarterly (e.g., \$1,000 annual maximum limited to $\$ 250$ per calendar quarter), a common practice in Medicare+Choice plans since 2001.

Nevertheless, the study by Cox and Henderson does highlight a subject that should concern health plan administrators 